\title{
The Role of Imaging in the Management of Cardiorenal Syndrome
}

\author{
Sajid Melvin George and Kambiz Kalantarinia \\ Division of Nephrology, University of Virginia Health System, P.O. Box 800133, Charlottesville, VA 22908, USA \\ Correspondence should be addressed to Kambiz Kalantarinia, kk6c@virginia.edu
}

Received 23 August 2010; Accepted 4 January 2011

Academic Editor: Claudio Ronco

Copyright ( 2011 S. M. George and K. Kalantarinia. This is an open access article distributed under the Creative Commons Attribution License, which permits unrestricted use, distribution, and reproduction in any medium, provided the original work is properly cited.

\begin{abstract}
Imaging of the kidney and the heart can provide valuable information in the diagnosis and management of cardiorenal syndromes. Ultrasound- (US-) based imaging (echocardiogram and renal US) is an essential component in the initial diagnostic workup of CRS. Echocardiography provides information on the structure and function of heart, and renal ultrasound is useful in differentiating between acute and chronic kidney disease and excluding certain causes of acute kidney injury such as obstructive uropathy. In this paper we overview the basic concepts of echocardiogram and renal ultrasound and will discuss the clinical utility of these imaging techniques in the management of cardiorenal syndromes. We will also discuss the role of other imaging modalities currently in clinical use such as computerized tomography and magnetic resonance imaging as well as novel techniques such as contrast-enhanced ultrasound imaging.
\end{abstract}

\section{Introduction}

The combination of cardiac and renal disease significantly increases the complexity and cost of health care [1]. The chronic kidney disease associated with heart failure has been recognized as an independent risk factor for morbidity and mortality [2-5]. The rate of cardiovascular mortality in the chronic kidney disease (CKD) population is 10-20 times that of those without CKD [6-8]. Recently, the Acute Dialysis Quality Initiative has published a document describing the definition and classification of cardiorenal syndrome (CRS) [1]. According to that document, the term cardiorenal syndrome refers to dysfunction of one organ system in presence of acute or chronic dysfunction of the other. According to the proposed classification, CRS is divided into 5 types. Types 1 and 2 include those with reduced kidney function due to either acute (type 1) or chronic cardiac dysfunction, and CRS 3 and 4 include worsening cardiac function in face of acute or chronic kidney disease, respectively. Secondary CRSs are grouped under type 5.

In this paper we will discuss the application of various imaging modalities in the diagnosis and management of CRS.

\section{Ultrasonography}

Imaging using ultrasound waves, ultrasonography, is a noninvasive, cost-effective, and widely available technology. The availability of new imaging modalities such as harmonic imaging, Doppler ultrasound for the study of blood flow, three-dimensional US and the advances in the technology and design of new transducers have significantly improved the quality of ultrasound (US) image. Currently, US imaging is the most widely used imaging modality in many fields, and indications for its use are expanding. US examination is considered the imaging modality of choice in the diagnosis and management of most cardiac and renal diseases. Therefore, the main focus of this paper would be on echocardiography and renal ultrasonography.

The US examination is based on interpretation of the character of the reflected sound waves from body tissues. The energy and the time lag at which these reflected US waves return and are picked up by the transducers determine the brightness and the depth of each tissue segment. The design and frequency of US transducers determine their utility in clinical imaging. In general, higher frequency probes generate images with higher resolution but because of limited 
tissue penetration, high frequency transducers are used for imaging of superficial structures. Imaging of the heart and the kidneys requires frequencies in the range of $2-5 \mathrm{MHz}$ (lower frequencies) to guarantee deeper penetration into the body. Imaging using ultrasound is limited in presence of gas and boney structures. High degree of reflection of US waves at the junction of soft tissue and gas or bone results in loss of signal from tissues beyond that point.

2.1. Echocardiography. Echocardiography is considered the preferred diagnostic method in cases suspicious of having heart failure [9]. It is a safe, noninvasive, and reproducible test applicable at bedside that provides valuable information on the anatomy and function of the heart. Using echocardiography, one can assess the structure of the myocardium and pericardium, global and regional LV function, and wall motion at rest and during pharmacologic stress. Twodimensional (2D), gray-scale, or B (for brightness) mode echocardiography provides real-time images of heart structures and their motion. By imaging the heart in different planes, information on the dimensions, surface area, and volume of different chambers of the heart and valves can be obtained. $\mathrm{M}$ (for motion) mode echocardiography image is the graphic representation of the movement of cardiac structures based on the 2D image. Doppler echocardiography and color flow imaging provides information on the blood flow velocities and the pattern of blood flow within the heart. Pulsed and continuous wave Doppler US provide information on the velocity of blood flow in a specific location or the whole area within the path of ultrasound, respectively. Color flow Doppler provides information on velocity, turbulence, and direction of blood flow in the form of a colorful image superimposed on a 2D image.

Echocardiography provides useful information on left ventricular (LV) function. These include LV ejection fraction (LVEF), stroke volume, cardiac index, fractional shortening and regional wall motion analysis, among others. LVEF is the fraction of the left ventricular volume at the end of diastole that is ejected at the end of each contraction. Although there are objective ways to measure LVEF by plugging in accurate measurements of LV dimensions or volumes into a formula, in most cases, LVEF is a visual estimation of LV function and its accuracy depends on the experience of the interpreter. In one study of individuals with heart failure and reduced LVEF 6 months after a myocardial infarction [10], the overall accuracy of echocardiography in correctly assessing radionuclide ventriculography LVEF was $86 \%$. In only one of 86 patients studied, there was a clinically significant difference between LVEFs estimated by the two methods (low LVEF by echocardiogram and normal LVEF by radionuclide technique) [10]. In another study, LVEF estimated by either echocardiography or electrocardiogram-gated single photon emission computed tomography (SPECT) were lower than those obtained by angiography. But they both did similarly well in accurately assessing LVEF of less than $40 \%$ or $35 \%$ in comparison to angiography [11]. Despite its limitations [12], determination of LVEF by 2-dimensional echocardiography is well accepted as the preferred measure of global LV function.
Certain echocardiographic parameters such as LV enddiastolic volume index, mitral deceleration time, and severity of mitral valve regurgitation are strong predictors of outcomes in individuals with advanced heart failure and reduced LVEF [13].

As many as $50 \%$ of all cases with clinical symptoms of heart failure have preserved LV function. Therefore, assessment of diastolic function in these cases is critical. Echocardiography is the noninvasive way of assessing diastolic function. Since almost $80 \%$ of LV filling during diastole (relaxation of the LV) occurs passively, abnormal relaxation of myocardium would result in impaired LV filling and diastolic dysfunction. M-mode, 2D, and Doppler echocardiogram are all useful in making the diagnosis of diastolic dysfunction and predicting outcomes [14]. Diastolic dysfunction can be graded based on the filling pressures and mitral inflow patterns obtained by echocardiography. Spectral tissue Doppler-derived index $\mathrm{E} / \mathrm{E}^{\prime}$ appears to be a valuable tool in assessing left atrial pressure [15]. E and $\mathrm{E}^{\prime}$ represent peak early diastolic mitral flow and mitral annulus velocities, respectively. This index has shown a linear relationship with LV diastolic pressure irrespective of LVEF, heart rhythm, and rate [15]. The diagnostic accuracy of $\mathrm{E} / \mathrm{E}^{\prime}$ is similar to that of B-type natriuretic peptide (BNP) in diagnosis of patients presenting with symptoms of heart failure with a wide range of LVEF [16].

Septal E/E' at a cut-off value of 13 in patients presenting with acute dyspnea and preserved systolic function has a sensitivity of $76 \%-82 \%$ and specificity of $88 \%-91 \%$ for diagnosis of heart failure $[17,18]$.

2.2. Renal Ultrasonography. Kidney size and the echogenecity of the renal parenchyma provide useful information in the workup of kidney diseases. Normal kidney measures $9-13 \mathrm{~cm}$ in long axis. Length of the kidney correlates with height of the person while kidney volume correlates with weight, body surface area, and height [19]. Small kidney size is consistent with chronic kidney disease (CKD). However, not all CKD cases are associated with small kidneys. Normal or enlarged kidneys are seen in cases with HIV-associated nephropathy (HIVAN) [20], diabetic nephropathy, and monoclonal gammapathies.

Within the renal cortex the back scatter of the US waves occurs mainly from the glomeruli and blood. In general, normal renal cortex generates an US image that is less echogenic or darker than the adjacent liver tissue at the same depth. Increased echogenecity of renal cortex, that is, same or brighter than the liver, indicates renal disease. This finding on the gray-scale US has a specificity of $96 \%$ and positive predictive value of $67 \%$ for diagnosis of kidney disease [21].

Since glomeruli occupy only $8 \%$ of the cortical volume, isolated glomerular disease will not produce increased echogenecity [22]. Increased cortical echogenecity is usually indicative of CKD. However, echogenic kidneys can also be seen in cases with acute kidney injury (AKI). As an example, large and swollen kidneys with increased echogenecity are reported in cases with acute tubular necrosis (ATN) or lupus nephritis [23-25]. 
Ultrasound imaging of the kidneys is very useful in the diagnosis of obstructive uropathy. In fact, a negative kidney US exam for hydronephrosis rules out obstruction as the cause of AKI. When dilatation of calyces and proximal ureter is found, the diagnosis of urinary tract obstruction can be made. However, dilatation of renal calyces alone does not always indicate urinary tract obstruction. In fact, caliceal dilatation can be found during pregnancy and also in cases with diabetes insipidus $[26,27]$. Dilatation of the distal ureter may provide clues for presence of obstruction at the level of bladder or urethra.

\section{Role of Ultrasound in Management of CRS Patients}

In patients suspicious of CRS, echocardiogram provides valuable information on cardiac structure and function and should be considered as one of the initial diagnostic studies. Around $27-40 \%$ of patients with acute decompensated heart failure (HF) develop acute kidney injury (AKI) [28]. At the same time, 45 to $63 \%$ of patients with chronic HF have CKD [29, 30]. Acute decompensated heart failure can occur in the setting of either systolic or diastolic dysfunction. Echocardiogram is the most useful diagnostic test in evaluating the cause of acute HF. In addition to providing information on systolic and diastolic function of the heart, echocardiogram is very useful in assessing regional wall motion abnormalities, condition and function of heart valves, and hemodynamics. Echocardiogram can also be used to rule out pericardial disease.

Systolic HF is defined as combination of symptoms of HF and LVEF less than 50\%. Heart Failure with normal LVEF, also referred to as diastolic dysfunction, was not recognized as an entity by the cardiology community until about two decades ago. Patients with HF and normal LVEF usually have normal or reduced LV size, but enlarged left atria [31]. Although it is thought that LVH is the cause of HF with normal LVEF, the criteria for its diagnosis are only met in less than $50 \%$ of cases with HF and normal EF. Instead, these individuals have increased LV mass-to-volume ratio [32]. Doppler echocardiogram can provide valuable information on LV relaxation, filling pressures, and stiffness. However, this information needs to be interpreted carefully with special attention to clinical presentation (acute versus chronic, presence of symptoms, blood pressure readings, etc.). The 2007 consensus statement of the Heart Failure and Echocardiography Associations of the European Society of Cardiology suggested 3 essential criteria for the diagnosis of diastolic heart failure or heart failure with normal ejection fraction. They are presence of signs and symptoms of HF, normal or mildly abnormal LV size and systolic function (LVEF $>50 \%$ and an $\mathrm{LV}$ end-diastolic volume $<97 \mathrm{~mL} / \mathrm{m}^{2}$ ), and evidence of LV diastolic dysfunction via invasive or noninvasive methods.

Renal ultrasonography is valuable in differentiating between acute and chronic kidney disease and ruling out obstruction as a cause of worsening renal function. In cases suspicious of type 1 CRS, a normal renal ultrasound examination is expected. This is due to the acute nature of condition and the fact that reduced GFR is resulting from renal hypoperfusion. An exception would be cases in which acute HF results in acute worsening of GFR from an already low baseline level, or acute on chronic KD. In these cases, US features of CKD do not rule out the possibility of CRS type 1. Availability of previous kidney ultrasound images for comparison would be of great value in these cases. A few small single center studies suggest a role for Doppler ultrasound in the differential diagnosis of AKI. In one study, a normal $(<0.71)$ resistive index [ (peak systolic velocity peak diastolic velocity)/peak diastolic velocity)] measured at the level of segmental renal arteries was seen in all individuals with a low fractional excretion of sodium (FENa), while individuals with high FENa had high resistive indices [33]. Among those with acute tubular necrosis and high FENa at baseline, there was a significant reduction in the RI after recovery from AKI episode [33].

\section{Other Imaging Studies}

4.1. Imaging of the Heart. Although echocardiogram is considered the imaging modality of choice in the workup of HF patients, in some cases more information on the structure and function of the heart might be necessary. In order to measure LVEF and volumes by echocardiography, a clear definition of endocardium is needed. This information is lacking in as many as $31 \%$ of cases [34]. Other imaging techniques might be needed to obtain more detailed information in these cases.

The most commonly used imaging in nuclear cardiology is single-photon emission computed tomography (SPECT) imaging of myocardial perfusion. After injection of the radiotracer, the isotope is extracted from the blood by viable myocytes and retained within the myocyte for some period of time. The photons are emitted from the myocardium which is in proportion to the magnitude of tracer uptake, and thus relates to perfusion. A gamma camera captures the photons and converts it into digital data which represents the magnitude of uptake and location of emission. Myocardial perfusion scanning using intravenous injection of technetium- $99 \mathrm{~m}-\left({ }^{99 \mathrm{~m}} \mathrm{Tc}-\right)$ labeled agents such as sestamibi ${ }^{99 \mathrm{~m}} \mathrm{Tc}$-labeled RBCs can be used for assessment of LVEF and regional wall motion abnormalities. Radionuclide imaging can be used to study the structure and function of the heart at rest and after exercise. LVEF, end-systolic, and end-diastolic volumes can be accurately measured by processing three dimensional images on gated SPECT using ${ }^{99 \mathrm{~m}} \mathrm{Tc}$ agents [9].

Radionuclide ventriculography can be used to assess the left ventricular function. It can be performed either as first pass or equilibrium-gated techniques. Both techniques provide reliable means to asses the left and right ventricular function. The equilibrium technique is referred to as multiple-gated acquisition (MUGA) scanning. The RVG technique has advantages over echocardiography as there are no assumptions made about the ventricular geometry. Thus radionuclide imaging can provide accurate data about ventricular function [35]. 
While there is a high degree of correlation between measurements of ventricular end-systolic and end-diastolic volumes measured by gated SPECT and cardiac MR imaging, it appears that cardiac MR is superior in providing more accurate chamber volumes $[36,37]$.

Positron emission tomography (PET) is used to assess myocardial viability by assessing its metabolism using FDG or myocardial perfusion using rubidium $(\mathrm{Rb})$ 82. PET imaging has several advantages over SPECT, including better spatial resolution, higher counting efficiencies, and excellent attenuation correction.

Computerized tomography (CT) techniques such as multi-slice CT and ultrafast electron beam tomography (EBT) are capable of providing images of the heart with high temporal and spatial resolutions. Multislice CT obtains images of the heart in many different imaging planes and is capable of providing information on cardiac volumes and dimensions. Fast imaging combined with electrocardiographic triggering reduces motion artifacts. EBT imaging occurs in milliseconds, which resolves the issue of cardiac motion during imaging. It has been shown to have comparable diagnostic accuracy in differentiating between ischemic and nonischemic cardiomyopathy compared to radionuclide stress testing in patients with HF [38]. However, EBT requires imaging using contrast for delineation of different chambers for determination of dimensions. This technique is also associated with high radiation exposure.

As a result of great advancements in the technology of magnetic resonance imaging, it can now be used for multiple purposes in evaluation of patients with cardiac diseases. Cardiac MR (CMR) is useful in assessing cardiac, great vessels and coronary anatomy and flow, ventricular function, myocardial viability, and perfusion. CMR provides the ability of imaging the heart in any desired plane in an unrestricted view, a clear advantage over echocardiography. Other advantages of CMR over echocardiography include the ease of studying the right ventricle due to its inherent threedimensional nature and superior border detection between ventricular blood pool and the myocardium [34, 39-41]. Cardiac MR has very high accuracy and reproducibility for determination of ventricular volumes, stroke volume and ejection fraction $[42,43]$. Using different MR sequences or techniques, it is possible to detect fibrosis, scarring, and inflammation of the myocardium. These advantages have resulted in making CMR the reference standard for ventricular volumetric assessment. In fact, in many institutions where this imaging modality is available, cardiac MR is considered the alternative diagnostic test to echocardiography in cases with poor ultrasound images or when myocarditis or infiltrative disease of the heart is suspected [44].

4.2. Imaging of the Kidney. Computerized tomography (CT) is the preferred imaging technique for the workup of kidney stones, renal masses, and renal arteries. Modern multidetector CT scanners are capable of acquiring thin slices of large areas of the body in one breath hold. Advancements in postprocessing techniques have also improved the diagnostic accuracy of CT scanning. Three-dimensional reconstruction of images is commonly used without compromising on the quality of the images. In fact, 3D reconstruction of CT angiograms has been rapidly omitting the need for catheter angiography $[45,46]$.

CT urography has almost completely replaced intravenous urograms in diagnosis of small stones and neoplasms of the kidney, ureters, and the urinary bladder. Filling defects within the collecting system might suggest a blood clot, stone, or a neoplasm. Unenhanced helical CT scan is the gold standard for diagnosing suspected renal colic secondary to kidney stones. Stones that are radiolucent on plain films are readily detectable by CT. In addition, CT scan provides valuable information on other signs of urinary tract obstruction such as hydronephrosis or hydroureter and streaking around these structures.

Contrast-enhanced CT is used to evaluate the arterial and venous supply of the kidney, renal parenchyma, and collecting system. Evaluation of the renal parenchyma occurs in the 3 phases, cortical phase, nephrographic, and excretory phase, after injection of the contrast. The detection of cortical or medullary lesions is best achieved during the nephrographic phase $[47,48]$. CT imaging can also be used as a functional imaging modality for applications such as estimation of the GFR. Of course the risk of contrast-induced nephropathy is one of the main drawbacks of contrastenhanced CT imaging, especially in individuals with acute or chronic kidney disease.

The preferred agent for radionuclide imaging of the kidneys is ${ }^{99 \mathrm{~m}} \mathrm{Tc}$. It provides good image quality with low radiation and has a short half-life. ${ }^{99 \mathrm{~m}} \mathrm{Tc}$ can be bound to diethylenetriaminepentaacetic acid (DTPA) for measurement of GFR or mercaptoacetyltriglycine (MAG3) to measure renal blood flow. This imaging modality can be used in the differential diagnosis of AKI. Kidney uptake of ${ }^{99 \mathrm{~m}} \mathrm{Tc}$ MAG3 in the first 1-2 minutes after injection is reduced in cases with acute tubular necrosis, while it would be normal in individuals with prerenal AKI. Renal uptake of the tracer during the late phase (20 minutes after injection) is expected to be high in prerenal AKI as well as ATN but low in postrenal AKI [49].

The main utility of MR imaging in the study of the genitourinary tract is in staging of malignancies. Magnetic resonance angiography may be used to study renal arteries, but its use is limited because of the risk of nephrogenic systemic fibrosis in individuals with reduced renal function [50]. This risk is especially high (up to 20\%) in individuals with AKI [51].

Sonographic imaging of kidneys with color Doppler and B mode has limited ability to evaluate the intrarenal arteries and arterioles. Imaging using ultrasound contrast agent can potentially overcome this limitation. The contrast agents used in renal US are gas-filled microbubbles that act similar to the red blood cells and remain in the intravascular space. In that regard, they are completely different from contrast-agents used in CT or MRI [52]. Contrast-enhanced echocardiography has been used in the clinical setting. A few US contrast agents are approved for the study of myocardial blood flow in humans. More recently, contrast enhanced ultrasonography (CEU) has been used in the study of changes in renal blood flow in response to physiologic and 
pharmacologic stimuli in animal models and in human subjects with promising results [53-60]. In some studies CEU has been superior to color and power Doppler US examination in determining the cause of AKI [61]. While B mode US can provide information on structure of the kidneys, addition of ultrasound contrast agents has the potential of providing additional information on the pattern and quantity of regional and total RBF [61]. Once approved for clinical use in humans, CEU may be of great value in the work up of cases suspicious of CRS since altered renal hemodynamics are thought to be the cause of reduced GFR in these cases.

\section{Conclusion}

Ultrasound based imaging of the heart (echocardiography) and the kidney are the methods of choice in the work up of cases suspicious of CRS. US imaging is safe, noninvasive and widely available. It provides valuable information on the structure and function of the heart and the structure of the kidney. In clinical scenarios in which echocardiography is not helpful, cardiac MR and radionuclide scintigraphy can be used to assess LV function and structure. CT scan of the kidneys and the urinary tract is useful in the diagnosis of urinary tract obstruction and kidney stones. Contrast-based CT and MR studies are of limited use in CRS because of the potential complications. Contrast-enhanced ultrasound and other new investigational imaging modalities (MR) may prove to be useful in management of CRS cases.

\section{References}

[1] C. Ronco, P. McCullough, S. D. Anker et al., "Cardio-renal syndromes: report from the consensus conference of the acute dialysis quality initiative," European Heart Journal, vol. 31, no. 6, pp. 703-711, 2010.

[2] K. Bibbins-Domingo, F. Lin, E. Vittinghoff, E. Barrett-Connor, D. Grady, and M. G. Shlipak, "Renal insufficiency as an independent predictor of mortality among women with heart failure," Journal of the American College of Cardiology, vol. 44, no. 8, pp. 1593-1600, 2004.

[3] A. G. Galil, H. S. Pinheiro, A. Chaoubah, D. M. Costa, and M. G. Bastos, "Chronic kidney disease increases cardiovascular unfavourable outcomes in outpatients with heart failure," BMC Nephrology, vol. 10, no. 1, article 31, 2009.

[4] S. Hamaguchi, M. Tsuchihashi-Makaya, S. Kinugawa et al., "Chronic kidney disease as an independent risk for long-term adverse outcomes in patients hospitalized with heart failure in Japan. Report from the Japanese Cardiac Registry of Heart Failure in Cardiology (JCARE-CARD)," Circulation Journal, vol. 73, no. 8, pp. 1442-1447, 2009.

[5] A. Ahmed, M. W. Rich, P. W. Sanders et al., "Chronic kidney disease associated mortality in diastolic versus systolic heart failure: a propensity matched study," American Journal of Cardiology, vol. 99, no. 3, pp. 393-398, 2007.

[6] A. K. Cheung, M. J. Sarnak, G. Yan et al., "Atherosclerotic cardiovascular disease risks in chronic hemodialysis patients," Kidney International, vol. 58, no. 1, pp. 353-362, 2000.

[7] A. K. Cheung, M. J. Sarnak, G. Yan et al., "Cardiac diseases in maintenance hemodialysis patients: results of the HEMO Study," Kidney International, vol. 65, no. 6, pp. 2380-2389, 2004.
[8] K. Hebert, A. Dias, M. C. Delgado et al., "Epidemiology and survival of the five stages of chronic kidney disease in a systolic heart failure population," European Journal of Heart Failure, vol. 12, no. 8, pp. 861-865, 2010.

[9] S. A.. Hunt, W. T. Abraham, M. H. Chin et al., "ACC/AHA Guideline Update for the Diagnosis and Management of Chronic Heart Failure in the Adult: a report of the American College of Cardiology/American Heart Association Task Force on Practice Guidelines (Writing Committee to Update the 2001 Guidelines for the Evaluation and Management of Heart Failure): developed in collaboration with the American College of Chest Physicians and the International Society for Heart and Lung Transplantation: endorsed by the Heart Rhythm Society," Circulation, vol. 112, pp. e154-e235, 2005.

[10] G. I. W. Galasko, S. Basu, A. Lahiri, and R. Senior, "Is echocardiography a valid tool to screen for left ventricular systolic dysfunction in chronic survivors of acute myocardial infarction? A comparison with radionuclide ventriculography," Heart, vol. 90, no. 12, pp. 1422-1426, 2004.

[11] D. E. Habash-Bseiso, R. Rokey, C. J. Berger, A. W. Weier, and P. O. H. Chyou, "Accuracy of noninvasive ejection fraction measurement in a large community-based clinic," Clinical medicine \& research., vol. 3, no. 2, pp. 75-82, 2005.

[12] J. L. Hare, J. K. Brown, and T. H. Marwick, "Performance of conventional echocardiographic parameters and myocardial measurements in the sequential evaluation of left ventricular function," American Journal of Cardiology, vol. 101, no. 5, pp. 706-711, 2008.

[13] P. A. Grayburn, C. P. Appleton, A. N. Demaria et al., "Echocardiographic predictors of morbidity and mortality in patients with advanced heart failure: the Beta-blocker Evaluation of Survival Trial (BEST)," Journal of the American College of Cardiology, vol. 45, no. 7, pp. 1064-1071, 2005.

[14] R. W. Troughton, D. L. Prior, C. M. Frampton et al., "Usefulness of tissue doppler and color M-mode indexes of left ventricular diastolic function in predicting outcomes in systolic left ventricular heart failure (from the ADEPT Study)," American Journal of Cardiology, vol. 96, no. 2, pp. 257-262, 2005.

[15] S. Arques, E. Roux, and R. Luccioni, "Current clinical applications of spectral tissue Doppler echocardiography (E/E' ratio) as a noninvasive surrogate for left ventricular diastolic pressures in the diagnosis of heart failure with preserved left ventricular systolic function," Cardiovascular Ultrasound, vol. 5, article 16, 2007.

[16] H. Dokainish, W. A. Zoghbi, N. M. Lakkis, M. A. Quinones, and S. F. Nagueh, "Comparative accuracy of B-type natriuretic peptide and tissue Doppler echocardiography in the diagnosis of congestive heart failure," American Journal of Cardiology, vol. 93, no. 9, pp. 1130-1135, 2004.

[17] S. Arques, E. Roux, P. Sbragia et al., "Accuracy of tissue Doppler echocardiography in the emergency diagnosis of decompensated heart failure with preserved left ventricular systolic function: comparison with B-type natriuretic peptide measurement," Echocardiography, vol. 22, no. 8, pp. 657-664, 2005.

[18] S. Arques, E. Roux, P. Sbragia et al., "Accuracy of tissue Doppler echocardiography in the diagnosis of new-onset congestive heart failure in patients with levels of B-type natriuretic peptide in the midrange and normal left ventricular ejection fraction," Echocardiography, vol. 23, no. 8, pp. 627-634, 2006. 
[19] S. A. Emamian, M. B. Nielsen, J. F. Pedersen, and L. Ytte, "Kidney dimensions at sonography: correlation with age, sex, and habitus in 665 adult volunteers," American Journal of Roentgenology, vol. 160, no. 1, pp. 83-86, 1993.

[20] R. M. Schaffer, G. E. Schwartz, and J. A. Becker, "Renal ultrasound in acquired immune deficiency syndrome," Radiology, vol. 153, no. 2, pp. 511-513, 1984.

[21] J. F. Platt, J. M. Rubin, R. A. Bowerman, and C. S. Marn, "The inability to detect kidney disease on the basis of echogenicity," American Journal of Roentgenology, vol. 151, no. 2, pp. 317319, 1988.

[22] E. Quaia and M. Bertolotto, "Renal parenchymal diseases: is characterization feasible with ultrasound?" European Radiology, vol. 12, no. 8, pp. 2006-2020, 2002.

[23] J. F. Platt, J. H. Ellis, J. M. Rubin, M. A. DiPietro, and A. B. Sedman, "Intrarenal arterial Doppler sonography in patients with nonobstructive renal disease. Correlation of resistive index with biopsy findings," American Journal of Roentgenology, vol. 154, no. 6, pp. 1223-1227, 1990.

[24] H. Esterbrook Longmaid, E. Rider, and J. Tymkiw, "Lupus nephritis. New sonographic findings," Journal of Ultrasound in Medicine, vol. 6, no. 2, pp. 75-79, 1987.

[25] A. T. Rosenfield, R. K. Zeman, D. V. Cicchetti, and N. J. Siegel, "Experimental acute tubular necrosis: US appearance," Radiology, vol. 157, no. 3, pp. 771-774, 1985.

[26] S. L. Peake, H. B. Roxburgh, and P. S. Le Langlois, "Ultrasonic assessment of hydronephrosis of pregnancy," Radiology, vol. 146, no. 1, pp. 167-170, 1983.

[27] J. Stevens, B. D. Brown, and J. P. McGahan, "Nephrogenic diabetes insipidus: a cause of severe nonobstructive urinary tract dilatation," Journal of Ultrasound in Medicine, vol. 14, no. 7, pp. 543-545, 1995.

[28] S. S. Gottlieb, W. Abraham, J. Butler et al., "The prognostic importance of different definitions of worsening renal function in congestive heart failure," Journal of Cardiac Failure, vol. 8, no. 3, pp. 136-141, 2002.

[29] J. T. Heywood, G. C. Fonarow, M. R. Costanzo, V. S. Mathur, J. R. Wigneswaran, and J. Wynne, "High prevalence of renal dysfunction and its impact on outcome in 118,465 patients hospitalized with acute decompensated heart failure: a report from the ADHERE database," Journal of Cardiac Failure, vol. 13, no. 6, pp. 422-430, 2007.

[30] A. Ahmed and R. C. Campbell, "Epidemiology of chronic kidney disease in heart failure," Heart Failure Clinics, vol. 4, no. 4, pp. 387-399, 2008.

[31] M. S. Maurer, D. L. King, L. El-Khoury Rumbarger, M. Packer, and D. Burkhoff, "Left heart failure with a normal ejection fraction: identification of different pathophysiologic mechanisms," Journal of Cardiac Failure, vol. 11, no. 3, pp. 177-187, 2005.

[32] D. W. Kitzman, W. C. Little, P. H. Brubaker et al., "Pathophysiological characterization of isolated diastolic heart failure in comparison to systolic heart failure," Journal of the American Medical Association, vol. 288, no. 17, pp. 2144-2150, 2002.

[33] M. Izumi, T. Sugiura, H. Nakamura, K. Nagatoya, E. Imai, and M. Hori, "Differential diagnosis of prerenal azotemia from acute tubular necrosis and prediction of recovery by Doppler ultrasound," American Journal of Kidney Diseases, vol. 35, no. 4, pp. 713-719, 2000.

[34] N. G. Bellenger, M. I. Burgess, S. G. Ray et al., "Comparison of left ventricular ejection fraction and volumes in heart failure by echocardiography, radionuclide ventriculography and cardiovascular magnetic resonance. Are they interchangeable?" European Heart Journal, vol. 21, no. 16, pp. 1387-1396, 2000.
[35] K. L. Lee, D. B. Pryor, K. S. Pieper et al., "Prognostic value of radionuclide angiography in medically treated patients with coronary artery disease. A comparison with clinical and catheterization variables," Circulation, vol. 82, no. 5, pp. 1705$1717,1990$.

[36] P. Sipola, K. Peuhkurinen, and E. Vanninen, "Comparison of gated single-photon emissioncomputed tomography with magnetic resonance imaging for evaluation of left ventricular volumes and ejection fraction in patients with idiopathic dilated cardiomyopathy," International Journal of Cardiovascular Imaging. In press.

[37] E. E. van derWall, A. J. H. A. Scholte, H. M. Siebelink, and J. J. Bax, "Assessment of left ventricular volumes; reliable by gated SPECT?" International Journal of Cardiovascular Imaging. In press.

[38] M. J. Budoff, B. Jacob, M. L. Rasouli, D. Yu, R. S. Chang, and D. M. Shavelle, "Comparison of electron beam computed tomography and technetium stress testing in differentiating cause of dilated versus ischemic cardiomyopathy," Journal of Computer Assisted Tomography, vol. 29, no. 5, pp. 699-703, 2005.

[39] K. Alfakih, S. Reid, T. Jones, and M. Sivananthan, "Assessment of ventricular function and mass by cardiac magnetic resonance imaging," European Radiology, vol. 14, no. 10, pp. 1813$1822,2004$.

[40] J. A. Utz, R. J. Herfkens, and J. A. Heinsimer, "Cine MR determination of left ventricular ejection fraction," American Journal of Roentgenology, vol. 148, no. 5, pp. 839-843, 1987.

[41] N. G. Bellenger, N. J. Marcus, C. Davies, M. Yacoub, N. R. Banner, and D. J. Pennell, "Left ventricular function and mass after orthotopic heart transplantation: a comparison of cardiovascular magnetic resonance with echocardiography," Journal of Heart and Lung Transplantation, vol. 19, no. 5, pp. 444-452, 2000.

[42] T. D. Karamitsos, L. E. Hudsmith, J. B. Selvanayagam, S. Neubauer, and J. M. Francis, "Operator induced variability in left ventricular measurements with cardiovascular magnetic resonance is improved after training," Journal of Cardiovascular Magnetic Resonance, vol. 9, no. 5, pp. 777-783, 2007.

[43] R. C. Semelka, E. Tomei, S. Wagner et al., "Interstudy reproducibility of dimensional and functional measurements between cine magnetic resonance studies in the morphologically abnormal left ventricle," American Heart Journal, vol. 119, no. 6, pp. 1367-1373, 1990.

[44] T. D. Karamitsos, J. M. Francis, S. Myerson, J. B. Selvanayagam, and S. Neubauer, "The role of cardiovascular magnetic resonance imaging in heart failure," Journal of the American College of Cardiology, vol. 54, no. 15, pp. 1407-1424, 2009.

[45] S. Read, C. Allen, and C. Hare, "Applications of computed tomography in renal imaging," Nephron, vol. 103, no. 2, pp. c29-c36, 2006.

[46] E. K. Fishman and L. P. Lawler, "CT angiography: principles, techniques and study optimization using 16-slice multidetector CT with isotropic datasets and 3D volume visualization," Critical Reviews in Computed Tomography, vol. 45, no. 5-6, pp. 355-388, 2004.

[47] B. A. Birnbaum, J. E. Jacobs, C. P. Langlotz, and P. Ramchandani, "Assessment of a bolus-tracking technique in helical renal CT to optimize nephrographic phase imaging," Radiology, vol. 211, no. 1, pp. 87-94, 1999.

[48] S. Sheth and E. K. Fishman, "Multi-detector row CT of the kidneys and urinary tract: techniques and applications in the diagnosis of benign diseases," Radiographics, vol. 24, no. 2, article e20, 2004. 
[49] S. E. Haufe, K. Riedmüller, and U. Haberkorn, "Nuclear medicine procedures for the diagnosis of acute and chronic renal failure," Nephron, vol. 103, no. 2, pp. c77-c84, 2006.

[50] S. Swaminathan and S. V. Shah, "New insights into nephrogenic systemic fibrosis," Journal of the American Society of Nephrology, vol. 18, no. 10, pp. 2636-2643, 2007.

[51] M. R. Prince, H. Zhang, M. Morris et al., "Incidence of nephrogenic systemic fibrosis at two large medical centers," Radiology, vol. 248, no. 3, pp. 807-816, 2008.

[52] R. A. O’Rourke, J. R. Lindner, and K. Wei, "Contrast echocardiography," Current Problems in Cardiology, vol. 27, no. 11, pp. 449-519, 2002.

[53] K. Kalantarinia, J. T. Belcik, J. T. Patrie, and K. Wei, "Realtime measurement of renal blood flow in healthy subjects using contrast-enhanced ultrasound," American Journal of Physiology, vol. 297, no. 4, pp. F1129-F1134, 2009.

[54] K. Wei, E. Le, J. P. Bin, M. Coggins, J. Thorpe, and S. Kaul, "Quantification of renal blood flow with contrast-enhanced ultrasound," Journal of the American College of Cardiology, vol. 37, no. 4, pp. 1135-1140, 2001.

[55] T. Schlosser, C. Pohl, C. Veltmann et al., "Feasibility of the flash-replenishment concept in renal tissue: which parameters affect the assessment of the contrast replenishment?" Ultrasound in Medicine and Biology, vol. 27, no. 7, pp. 937-944, 2001.

[56] Y. Hosotani, N. Takahashi, H. Kiyomoto et al., "A new method for evaluation of split renal cortical blood flow with contrast echography," Hypertension Research, vol. 25, no. 1, pp. 77-83, 2002.

[57] N. Kishimoto, Y. Mori, T. Nishiue et al., "Ultrasound evaluation of valsartan therapy for renal cortical perfusion," Hypertension Research, vol. 27, no. 5, pp. 345-349, 2004.

[58] F. Lefèvre, J. M. Correas, S. Briançon, O. Hélénon, M. Kessler, and M. Claudon, "Contrast-enhanced sonography of the renal transplant using triggered pulse-inversion imaging: preliminary results," Ultrasound in Medicine and Biology, vol. 28, no. 3, pp. 303-314, 2002.

[59] R. Lencioni, S. Pinto, D. Cioni, and C. Bartolozzi, "Contrastenhanced doppler ultrasound of renal artery stenosis: prologue to a promising future," Echocardiography, vol. 16, no. 7, pp. 767-773, 1999.

[60] S. M. Gruenewald, T. Huster, G. Larcos, D. C. Farlow, K. K. L. Choong, and Y. Mudaliar, "Acute renal failure in critically ill patients: evaluation of an ultrasound contrast agent," Australasian Radiology, vol. 43, no. 2, pp. 180-184, 1999.

[61] J. M. Correas, M. Claudon, F. Tranquart, and O. Hélénon, "The kidney: imaging with microbubble contrast agents," Ultrasound Quarterly, vol. 22, no. 1, pp. 53-66, 2006. 


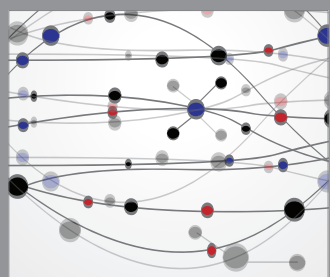

The Scientific World Journal
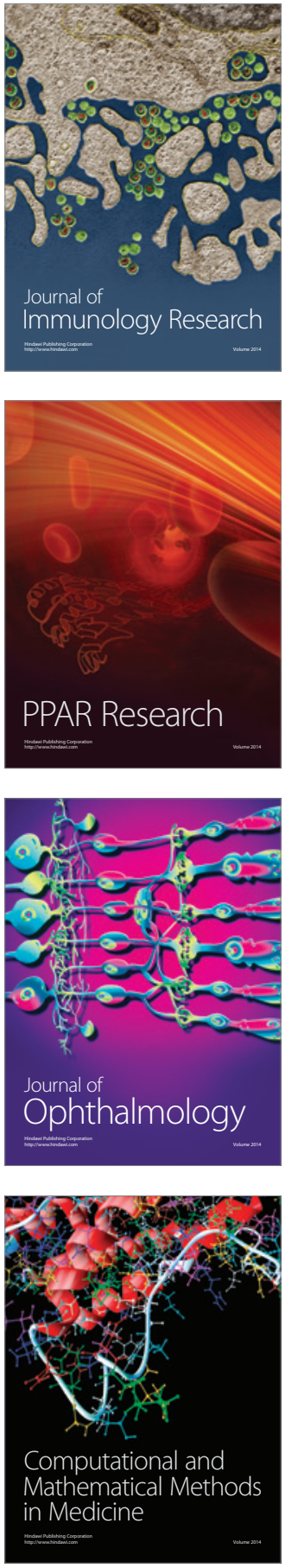

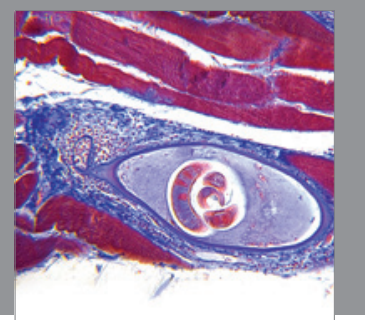

Gastroenterology

Research and Practice
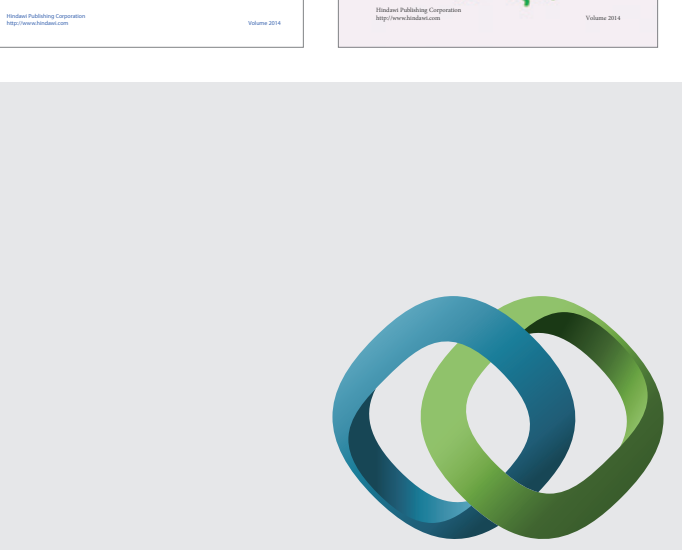

\section{Hindawi}

Submit your manuscripts at

http://www.hindawi.com
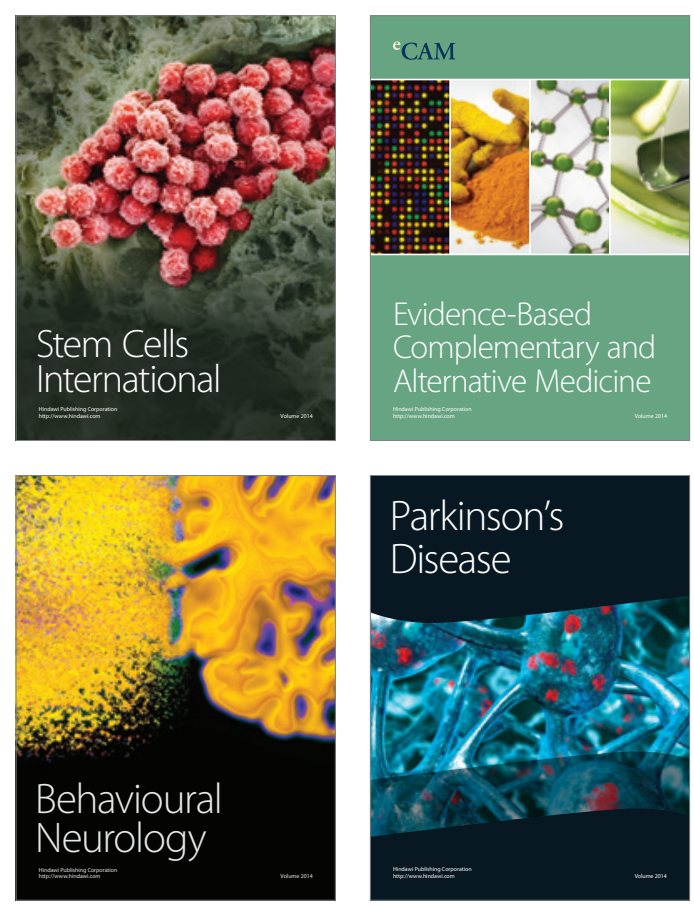

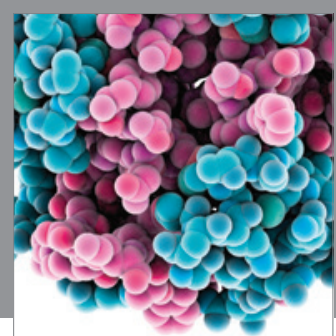

Journal of
Diabetes Research

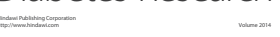

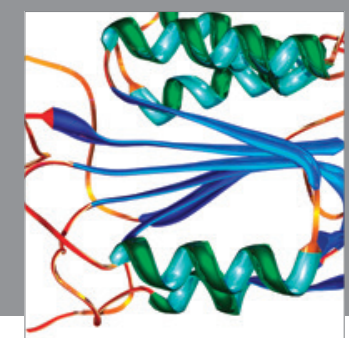

Disease Markers
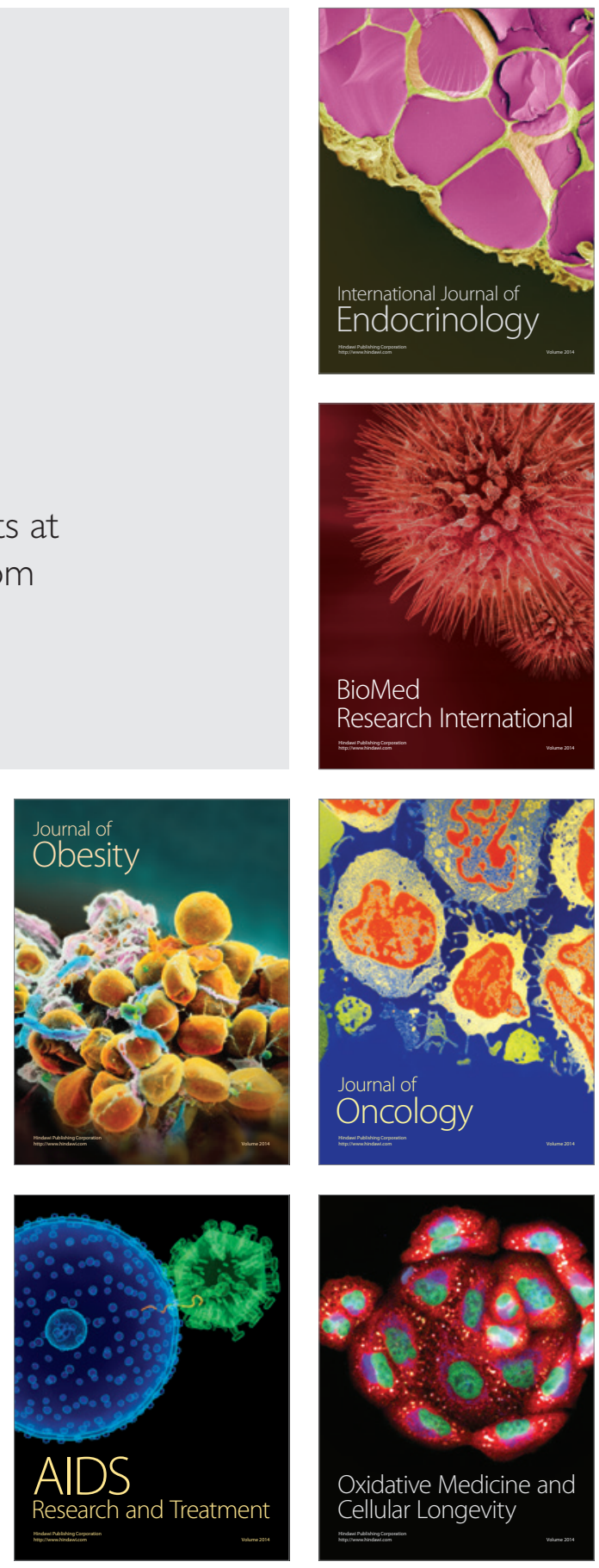